Vol. 83 No. 3 September 2018

\title{
CYTOLOGIA
}

International Journal of Cytogenetics and Cell Biology 


\title{
Technical Note:
}

\section{Development of a Misrepair Visualization Assay in the Live Embryo of Oryzias latipes by Partial Duplication System}

\author{
Tomomi Watanabe-Asaka, Shoji Oda and Hiroshi Mitani* \\ Graduate School of Frontier Sciences, The University of Tokyo, 5-1-5 Kashiwanoha, Kashiwa, Chiba 277-8562, Japan
}

Received June 9, 2018; accepted July 9, 2018

Radiation and environmental substances affect all cells in the tissue composing the body. The method using gene modification such as transgenic technology enables the measurement of the mutation risk in both somatic and germ cells without losing the tissue structure by cell death or dissection. Recently, an in vivo system has been reported to detect reversion mutations in adult mice using a partial overlapping sequence of murine HPRT gene locus (Noda et al. 2015). However, few methods have been established to evaluate influence of environmental stresses on an individual cell of the body especially in developmental stages. In this study, we established a reversion detection system with shorter (a few hundred bp) homology sequence using medaka (Oryzias latipes), which develops outside the maternal body with transparent body.

Medaka beta-actin promoter (olßactin pro, $3.1 \mathrm{~kb}$ ) induces strong gene expression in many somatic cells (Hamada et al. 1998). As an indicator of the reversion, $680 \mathrm{bp}$ of the $3^{\prime}$ region of olßactin pro, EGFP coding sequence and $S V 40$ polyA were inserted downstream of the full length olßactin pro::mCherry-bGH polyA construct. Somatic cells expressing mCherry, which the construct was introduced into the cell, changed to GFP expression after a reversion mutation in the region of partially duplicated olßactin pro. Therefore, EGFP expression was regarded as a result of reversion mutation in living cells caused by DNA damage and followed by olßactin pro::EGFP-SV40 polyA construction.

The plasmid vector was microinjected into the fertilized eggs of medaka embryo and 5 Gy of $\gamma$-ray irradia- tion around the blastula and gastrula stage when mCherry has already expressed in the cells like a mosaic. The image of the hatching stage embryo expressing GFP and mCherry was obtained by an upright microscopy (BX-50, Olympus, Tokyo, Japan) with excitation 488 and $584 \mathrm{~nm}$ and a digital steal camera (DFC7000T, Leica microsystems GmbH, Wetzlar, Germany). Images were processed with LAS v4.8 (Leica microsystems) to create maximum-intensity projection images.

Three out of 12 embryos expressed GFP in single or some cells after the irradiation, which is hardly observed in the control, indicating that revertant detection worked in vivo. This method will be useful to analyze reversion mutation during development without any dissections and tissue reconstruction. It is possible to evaluate the risk of somatic cells and germline mutations caused by radiation and environmental substances on tissue stem cells in vivo using this technique.

\section{References}

Hamada, K., Tamaki, K., Sasado, T., Watai, Y., Kani, S., Wakamatsu, Y., Ozato, K., Kinoshita, M., Kohno, R., Takagi, S. and Kimura, M. 1998. Usefulness of the medaka beta-actin promoter investigated using a mutant GFP reporter gene in transgenic medaka (Oryzias latipes). Mol. Mar. Biol. Biotechnol. 3: 173-180.

Noda, A., Suemori, H., Hirai, Y., Hamasaki, K., Kodama, Y., Mitani, H., Landes, D. R. and Nakamura, N. 2015. Creation of mice bearing a partial duplication of HPRT gene marked with a GFP gene and detection of revertant cells in situ as GFP-positive somatic cells. PLoS ONE 10: e0136041. 